\title{
Osteoporotic bone microstructure by collagenase etching
}

I G MACKIE, ${ }^{1}$ M GREEN ${ }^{2}$ H CLARKE ${ }^{2}$ AND D H ISAAC ${ }^{3}$

From the ${ }^{1}$ Department of Traumatic and Orthopaedic Surgery, UWCM, Cardiff Royal Infirmary, Cardiff; ${ }^{2}$ MEMS, UWIST, PO Box 25, Cardiff; and the ${ }^{3}$ Department of Materials Engineering, University of Wales, Swansea

SUMmARY Collagenase etching has been used to show the microstructure of bone from patients suffering from primary osteoporosis. Both polished and unpolished surfaces of trabecular bone from femoral heads were treated with collagenase solution before study in the scanning electron microscope. The polished surfaces show the mineral component of this bone as small rounded $\bigcirc$ units $\sim 10-20 \mathrm{~nm}$ across, which aggregate to form a continuous phase of contiguous spheroidal $\vec{\sim}$ particles $\sim 100 \mathrm{~nm}$ across. Lamellations are clearly seen to be due to the removal of collagen fibres up to $\sim 200 \mathrm{~nm}$ across, fibres in adjacent lamellae being arranged approximately perpendicular to each other. The unpolished surfaces also show small rounded units, which $\vec{\emptyset}$ aggregate into rods of mineral $\sim 100 \mathrm{~nm}$ across. Although these rods form a connected system, $\emptyset^{\infty}$ they are loosely packed, compatible with their being interspersed with the collagen fibres in vivo. This model for the detailed microstructure of bone is consistent with specimens from a number of other sources and shows no features unique to osteoporosis.

There are many published studies demonstrating the reduction in trabecular bone volume and bone mineral content in osteoporosis. ${ }^{1-5}$ The ultrastructure of the mineral and its relation with the collagen, however, have received little attention.

The structure of the mineral component of normal bone material has long been the subject of investigation, giving rise to a diversity of opinion. Early investigators, relying on line broadening and low angle diffraction experiments ${ }^{6}$ and transmission electron microscopy of thinly sectioned material, ${ }^{7-9}$ have suggested the presence of a variety of needle and plate shaped hydroxyapatite crystals with dimensions varying between $\sim 2 \mathrm{~nm}$ and $150 \mathrm{~nm}$. Recent experiments on the disaggregation of bone from a variety of sources have been interpreted as resulting from hydroxyapatite crystals in a tabular or book-like form $\sim 45 \mathrm{~nm}$ long by $30 \mathrm{~nm}$ wide. ${ }^{10}$

On the other hand, a number of contemporary investigators have proposed models in which spheroidal shaped particles $\sim 100 \mathrm{~nm}$ in diameter aggregate to form the mineral component of macroscopic bone. Boyde observed such 'spheroidal particles' in

Accepted for publication 1 October 1988.

Correspondence to Dr D H Isaac, Department of Materials Engineering, University of Wales, Swansea SA2 8PP. fetal bone ${ }^{11}$ and isolated similar particles from solutions of $\mathrm{NaOCl}$ used to wash bone and dentine. ${ }^{12}$ Sela reported observing 'calcospherites' $100-300 \mathrm{~nm}$ in diameter in osteogenic tumours. ${ }^{13}$ Pautard isolated similar particles from 6 day old mouse calverial bone. ${ }^{14}$ Contiguous spheroidal particles have also been noted in bovine and human compact lamellar bone following ion bombardment. ${ }^{15}{ }^{16}$ Criticism that the techniques used for the investigation of the structure of bone mineral have damaged the mineral and hence produced artefacts led us to employ a more subtle, biochemical etching technique involving the incubation of both polished and unprocessed bone surfaces in a solution of $\widetilde{N}$ collagenase. Collagenase etching has been success- $N$ fully used in this laboratory to demonstrate the N structure of bovine bone,,$^{13}$ fish and chicken $\sigma$ bone, ${ }^{19}{ }^{20}$ human bone of archaeological interest, ${ }^{21}$ and human bone free from disorders. ${ }^{22}$ Here we report its application to osteoporotic human bone.

\section{Materials and methods}

Samples of trabecular bone were obtained from 16 female patients aged 58-92. They were all admitted to the casualty unit following fracture of the neck of the femur. The patients were diagnosed as 
osteoporotic on the basis of radiographic and histological examination. ${ }^{5}$ They subsequently underwent total hip replacement.

The intact femoral heads were sectioned with a hand hacksaw in the mediolateral plane. Specimens were then removed from the centre of the head and prepared for examination in the scanning electron microscope.
Specimens were first polished using carbide papers and polishing alumina. Subsequently they were thoroughly washed in distilled water to remove any adherent alumina, then boiled in distilled water for five minutes to denature the collagen. Aliquots $(5 \mathrm{ml})$ of collagenase solution were made up in a calcium containing trometamol (TRIS) buffer ( $\mathrm{pH}$ 7.4) containing 2100 units of collagenase. Each

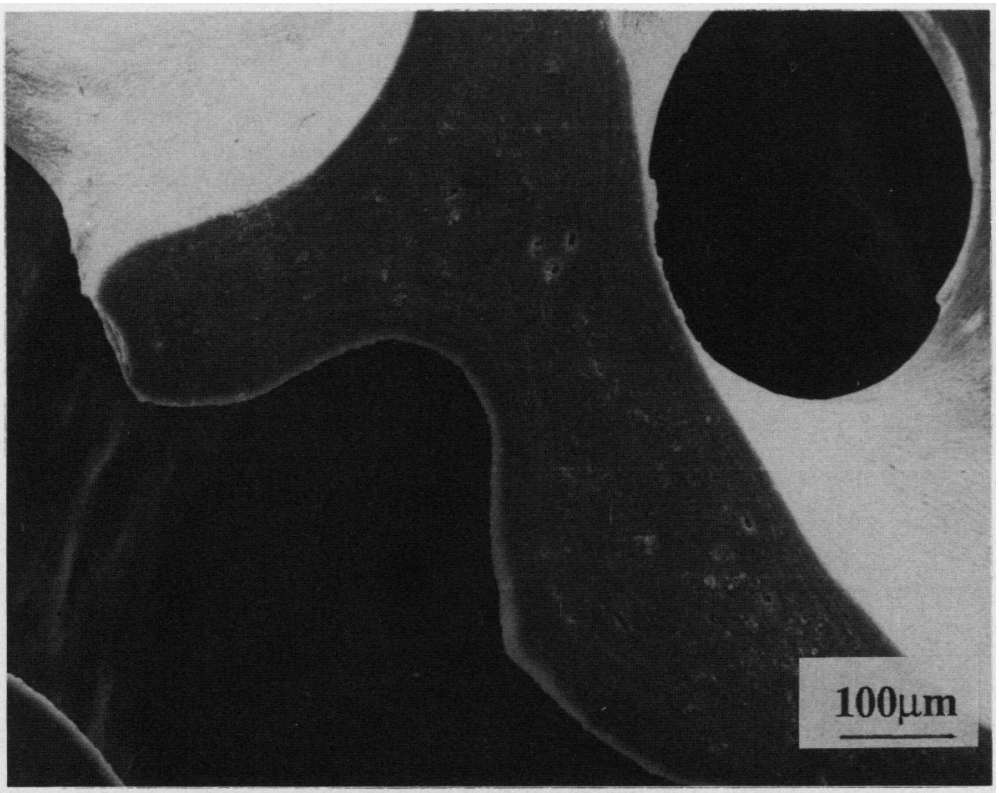

Fig. 1 Low magnification scanning electron micrograph of collagenase etched osteoporotic trabecular bone. The central area has been polished and shows some lamellation and in the background are unpolished surfaces.

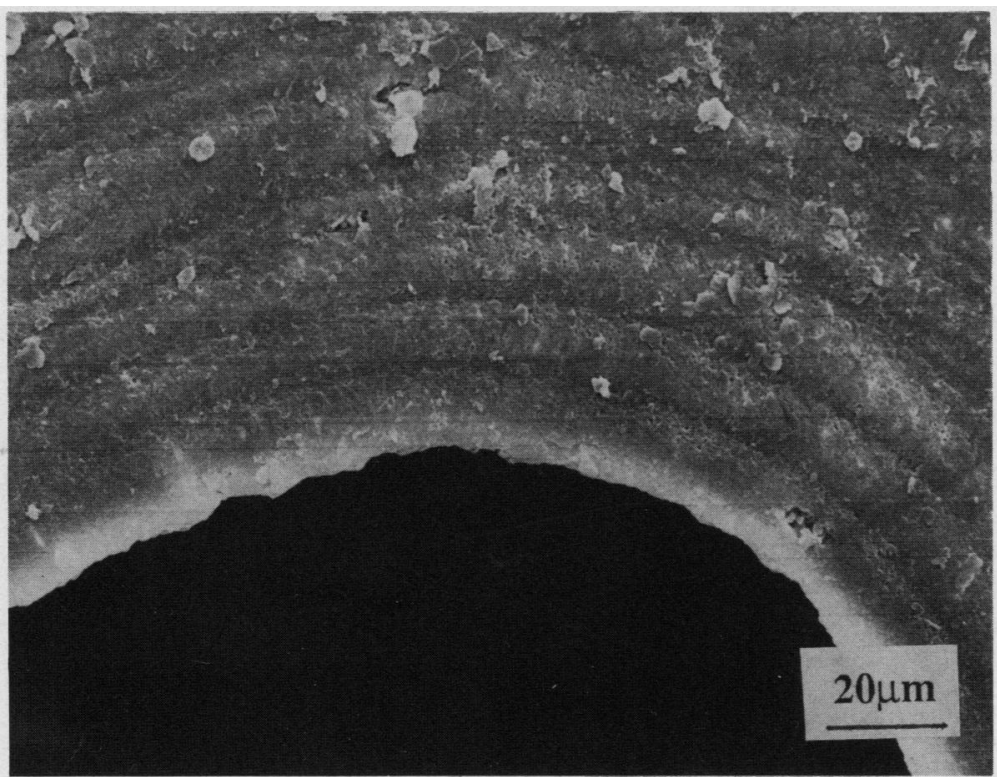

Fig. 2 Higher magnification of the polished surface of Fig. 1 showing the lamellar structure more clearly. 


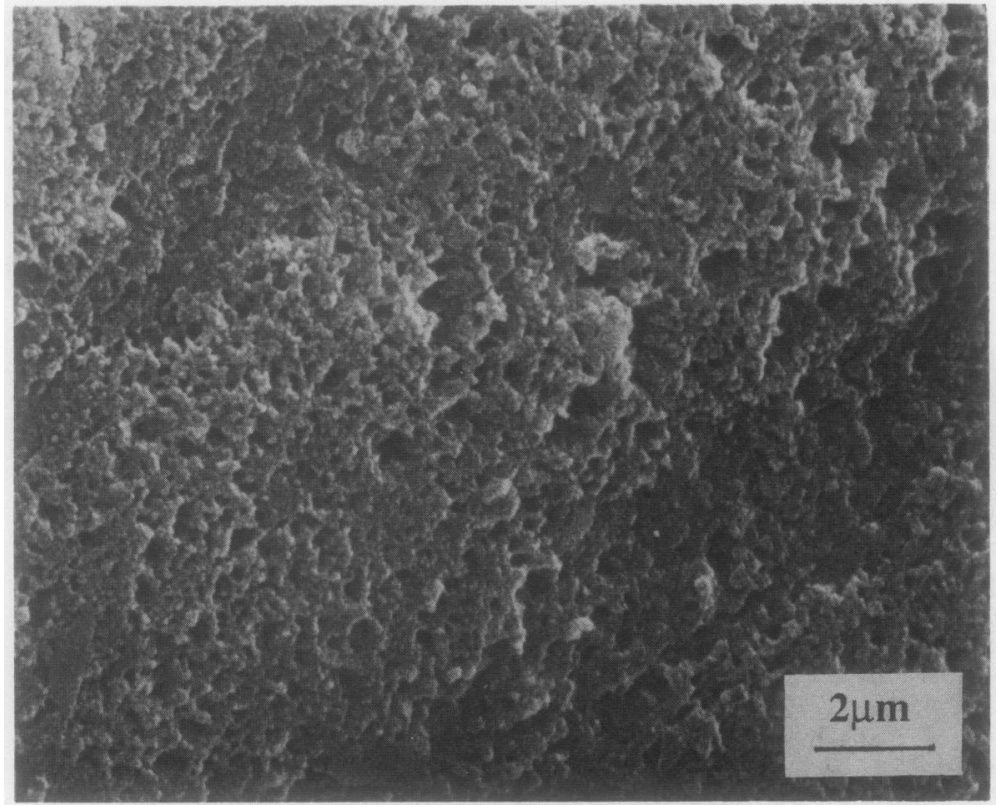

Fig. 3 More detailed micrograph showing the origins of the lamellar structure. Light lamellae include large numbers of holes from which collagen fibres up to $\sim 200 \mathrm{~nm}$ across running approximately perpendicular to the surface have been removed, whereas the dark lamellae have few such holes.

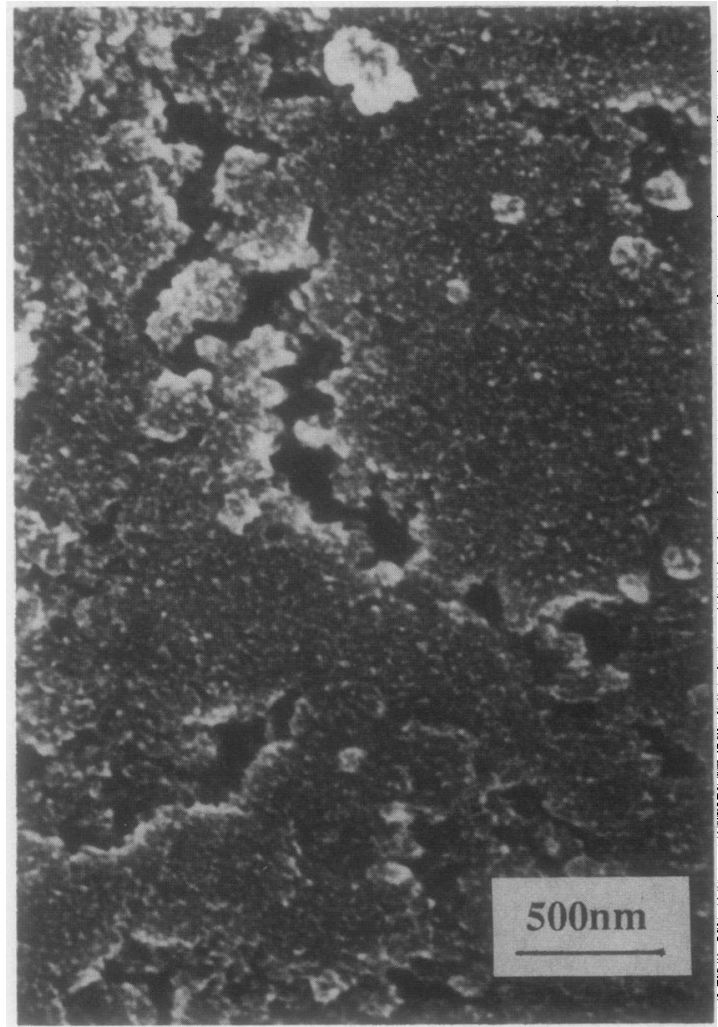

Fig. 4 The polished collagenase etched surface of osteoporotic trabecular bone at much higher resolution shows spheroidal shaped units $\sim 100 \mathrm{~nm}$ across.

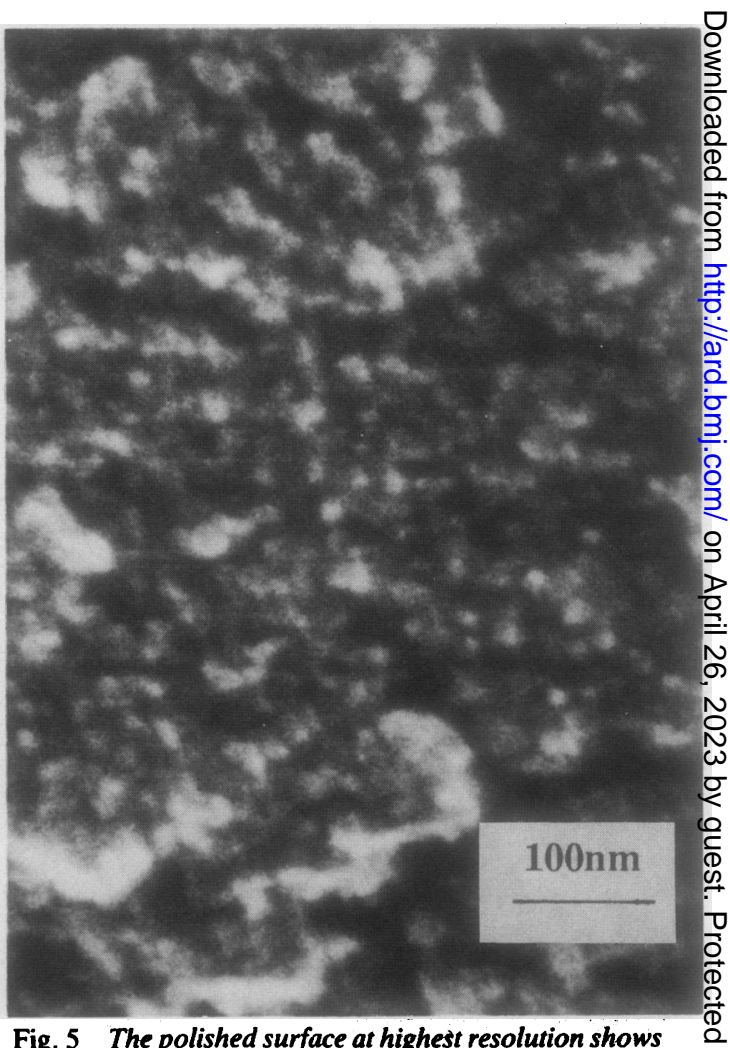

Fig. 5 The polished surface at highest resolution shows that the basic building blocks for the mineral component are small rounded units $\sim 10-20 \mathrm{~nm}$ across. 


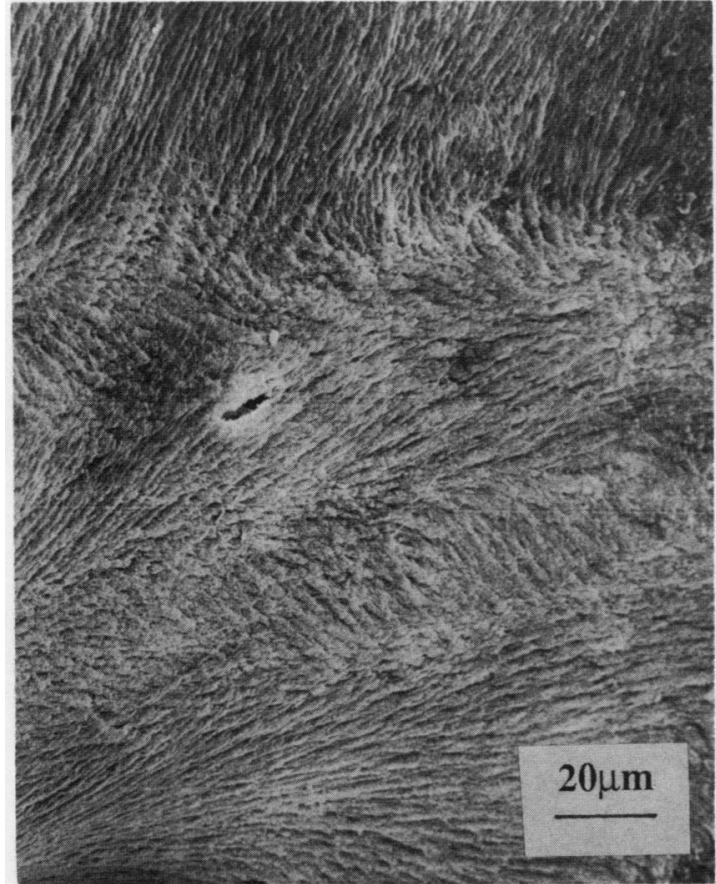

Fig. 6 The unpolished surface of collagenase treated osteoporotic trabecular bone. Several osteocytic lacunae are seen and the mineral forms a pattern suggesting rods of varying preferred orientation.

specimen was bathed in one aliquot at $37^{\circ} \mathrm{C}$ for one week to ensure adequate etching. After incubation the specimens were coated with gold and examined in the scanning electron microscope. The low magnification photographs of Figs 1, 2, 3, 6, and 7 were taken in a Jeol $35 \mathrm{C}$ operating at $30 \mathrm{kV}$. The higher resolution micrographs of Figs 4, 5, 8, and 9 were obtained on a Jeol $120 \mathrm{C}$ Temscan operating in the scanning mode, at an accelerating voltage of 100 $\mathrm{kV}$.

\section{Results}

Figure 1 is a low magnification electron micrograph of a polished and collagenase etched section through trabecular bone. The central polished surface is evident, as are the unpolished areas beyond. Closer examination of the polished area shows lamellation, which appears as alternate light and dark areas $\sim 10$ $\mu \mathrm{m}$ wide. This lamellation can be more clearly observed in the higher magnification micrographs of Figs 2 and 3. It can be seen that the light layers show a large number of holes up to $\sim 200 \mathrm{~nm}$ in diameter.

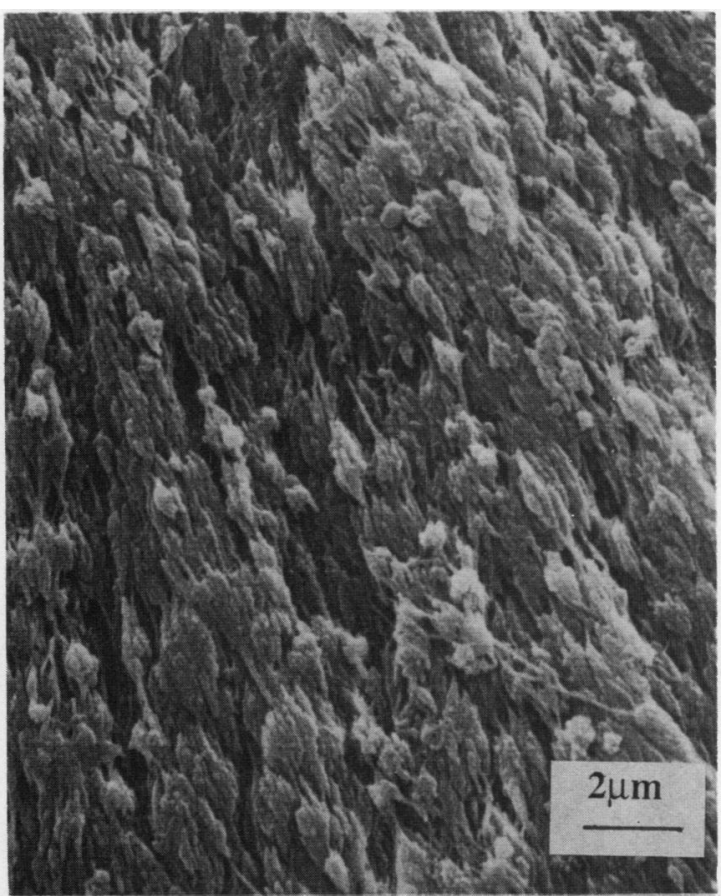

Fig. 7 A higher magnification micrograph of the unpolished surface showing the mineral as loosely packed rods with numerous interconnections, forming a continuous mineral phase.

These holes indicate the in vivo position of the collagen, which exists in the form of fibres of up to this diameter. The mineral component is shown to be a continuous phase surrounding the holes vacated by the collagen. The mineral phase consists of contiguous spheroids $\sim 100 \mathrm{~nm}$ across. At higher magnification (Fig. 4) these spheroids are seen to be composed of smaller units. In the high resolution picture of Fig. 5 these smaller units are observed as rounded particles $\sim 10-20 \mathrm{~nm}$ in diameter. At this resolution micrographs of the light and dark areas look essentially the same.

Figure 6 is a low magnification micrograph of an unpolished surface of a collagenase treated trabecula. Osteocytic lacunae are evident, and the pattern of the mineral can be observed to vary over the bone surface. On closer examination (Fig. 7) the hydroxyapatite is seen to be in the form of rods, which are interconnected forming the continuous mineral phase. The structure is not tightly packed but shows clearly the areas from which collagen fibres have been removed. At yet higher magnification (Fig. 8) these rods can be seen to be $\sim 100 \mathrm{~nm}$ in 


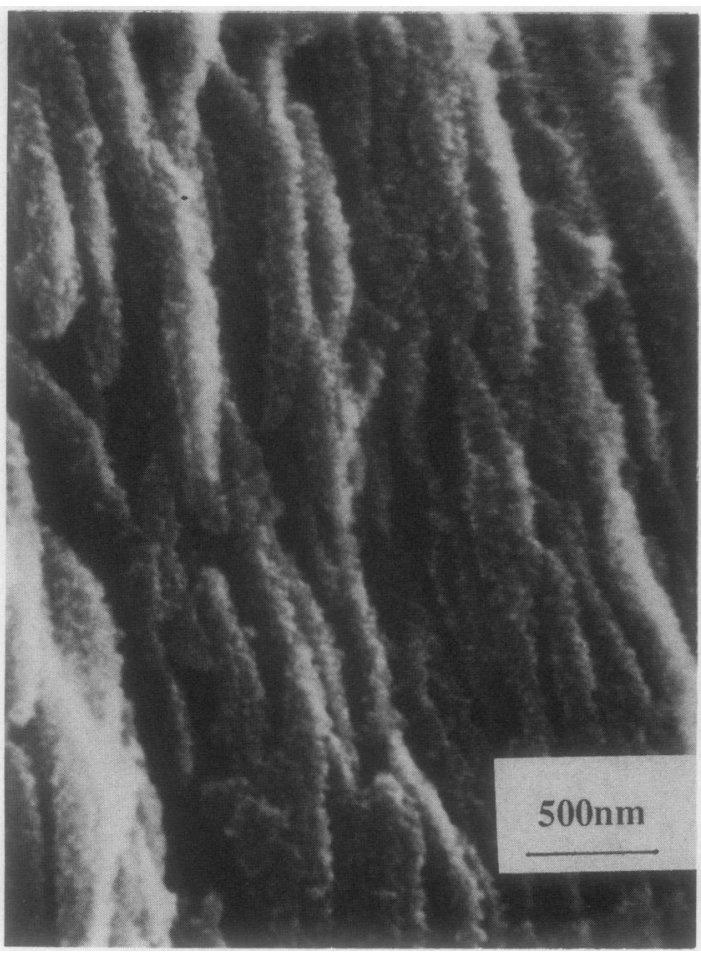

Fig. 8 The unpolished surface of collagenase etched osteoporotic trabecular bone at high magnification shows a complex plexiform of mineral rods and suggests that the mineral rods are composed of still smaller units.

diameter, and in the very high resolution micrograph (Fig. 9) the ultimate units are visualised as the same type of rounded particles $(\sim 10-20 \mathrm{~nm}$ across) as observed in the polished sections (Fig. 5).

\section{Discussion}

The classical model for the structure of bone mineral and its relation with the collagen envisages a mineral phase consiting of discrete needle or plate-like crystallites intimately linked with the tropocollagen molecules. Recent investigations in this laboratory ${ }^{17-22}$ have proposed a model for bone in which the mineral exists in the form of a continuous phase composed of spheroidal particles of $\sim 100 \mathrm{~nm}$ in diameter, which are themselves made up of smaller units $\sim 10-20 \mathrm{~nm}$ across.

This latter model is more readily reconciled with the known mechanical properties of bone than the former classical model, in which the mineral is said merely to stiffen the collagen. The failure of this model becomes apparent if a bone which has been

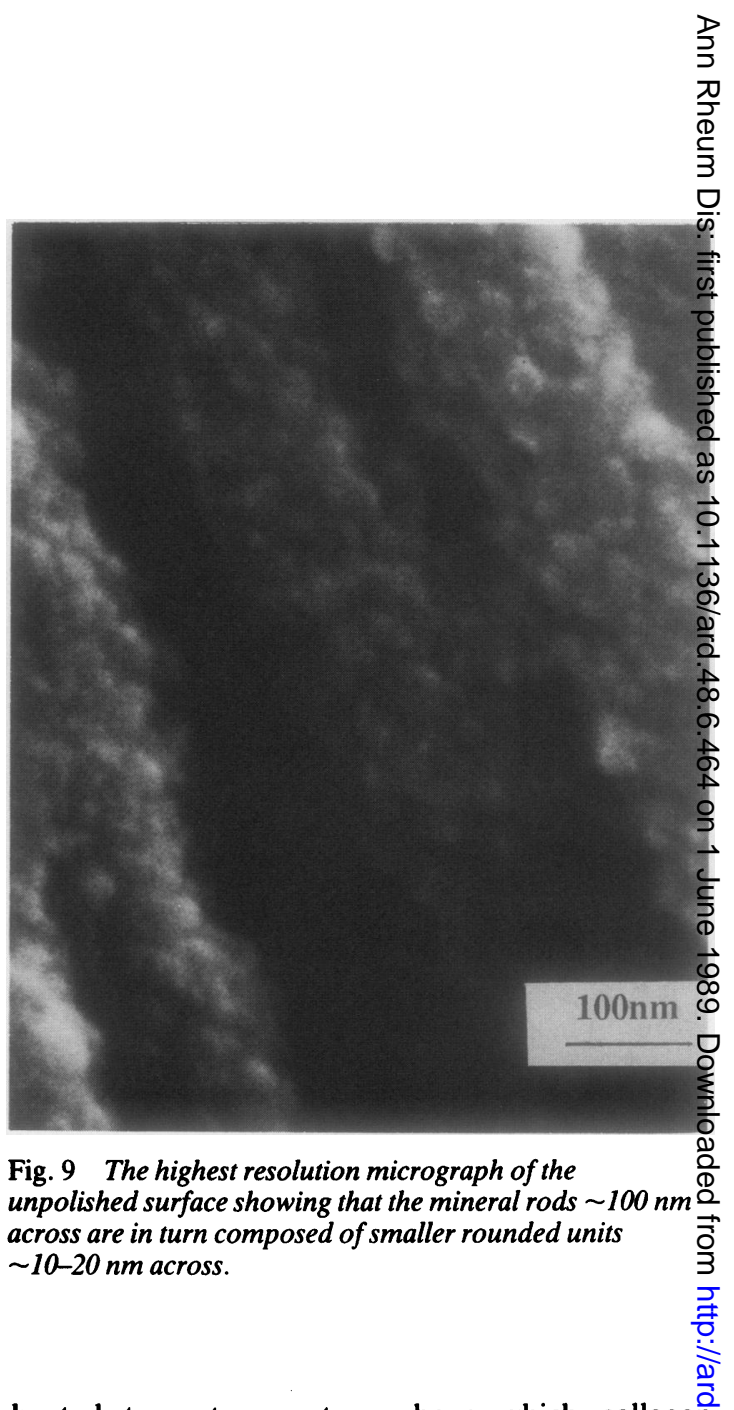

heated to a temperature above which collage denatures is subjected to mechanical testing. If the. mineral merely stiffened the collagen one would expect the denaturation of the collagen to totally distrupt the mechanical integrity of the bone. This however, clearly does not occur.

The holes observed in the mineral phase aro produced by the removal of the collagen owing to the action of collagenase, and therefore indicate tho in vivo position and direction of the collagen fibresin The lamellation observed in the polished sections is reminiscent of the lamellar structure of bovine secondary osteons, which have been studied in some detail using the same technique. ${ }^{17} 18$ It has beef shown that the lamellation in bovine secondar? osteons is a result of the direction of the collagen fibre orientation changing through approximately 980 degrees from one lamella to the next. ${ }^{18}$ Althoug such detailed examination has not been carried out on this human material, it is reasonable to assume that the structural basis for the lamellation is similare

It appears from examination of micrographs of 
the unpolished surfaces (Figs 8 and 9) of this osteoporotic bone that the mineral phase is in the form of columns with the collagen interdispersed between them. This is compatible with the polished sections through trabeculae (Figs 4 and 5), in which the mineral is observed to form a continuous phase around the collagen fibres. All these pictures are directly equivalent to those previously obtained on normal human trabecular bone from patients with no known bone disorders. ${ }^{22}$

The results of this investigation of the microstructure of trabecular bone derived from patients suffering from primary osteoporosis support the more recent model for the structure of bone. The detailed mineral structure is similar to that observed in bovine bone, ${ }^{17}{ }^{18}$ fish bone, ${ }^{19}$ chicken bone, ${ }^{20}$ and normal human bone. ${ }^{22}$

\section{Conclusions}

Bone mineral obtained from patients suffering from primary osteoporosis was found to exist as a continuous phase consisting of columns of mineral interdispersed with collagen. The mineral columns are $\sim 100 \mathrm{~nm}$ in diameter and appear to be of roughly circular section. They are composed of an ordered arrangement of smaller units $\sim 10-20 \mathrm{~nm}$ in diameter, which appear to be the basic building blocks of bone mineral. There is no apparent difference between normal and porotic bone at this ultrastructural level.

This work was supported by a grant from the Arthritis and Rheumatism Council for research. The authors wish to express their gratitude to the Trustee Savings Bank, who kindly donated the funds to make the grant possible.

\section{References}

1 Podenphant J, Herss Nielsen V-A, Riis B J, Gotfredsen A, Christiansen $C$. Bone mass, bone structure and vertebral fractures in osteoporotic patients. Bone 1987; 8: 127-30.

2 Parfitt A M. Age related structural changes in trabecular and cortical bone: cellular mechanisms and biomechanical consequences. Calcif Tissue Int 1984; 36: S123-8.

3 Burkhardt R, Kettner G, Bohm W, et al. Changes in trabecular bone, haematopoiesis and bone marrow vessels in aplastic anemia, primary osteoporosis and old age: a comparative histomorphometric study. Bone 1987; 8: 157-64.

4 Firooznia H, Rafii M, Golimbu C. Schwartz M S. Clinical utility of bone mineral content measurement in the diagnosis and treatment of osteoporosis. NY State J Med 1986: 626-9.

5 Recker R R, ed. Bone histomorphometry: techniques and interpretation. Cleveland: CRC Press, 1983.

6 Engstrom A. Aspects of the molecular structure of bone. In: Bourne G H, ed. The biochemistry and physiology of bone. 2nd ed. Vol 1. New York: Academic Press, 1972: 237-58.

7 Cameron D A. The ultrastructure of bone. In: Bourne G H, ed. The biochemistry and physiology of bone. 2nd ed. Vol 1. New York: Academic Press, 1972: 191-236.

8 Jackson S A, Cartwright A G, Lewis D. The morphology of bone mineral crystals. Calcified Tissue Research 1978; 25: 217-22.

9 Bocciarelli D S. Morphology of crystallites in bone. Calcified Tissue Research 1970; 5: 261-9.

10 Weiner S, Price A. Disaggregation of bone into crystals. Calcif Tissue Int 1986; 39: 365-75.

11 Boyde A. Scanning electron microscope studies of bone. In: Bourne G H, ed. The biochemistry and physiology of bone. 2nd ed. Vol 1. New York: Academic Press, 1972: 259-310.

12 Boyde A. Sela J. Scanning electron microscope study of separated calcospherites from the matrices of different mineralising systems. Calcified Tissue Research 1978; 26: 47-9.

13 Sela J. Bone remodelling in pathologic conditions. Calcified Tissue Research 1977; 23: 229-34.

14 Pautard F G E. Phosphorus and bone. In: Williams R J P, DuSilva J F, eds. New trends in bioinorganic chemistry. New York: Academic Press, 1978: 261-354.

15 Turner I G, Jenkins G M. The spatial arrangement of bone mineral as revealed by ion bombardment. Biomaterials 1981; 2: 234-8.

16 Turner I G. A study of the spatial organisation of the mineral component of bone. Swansea: University College of Swansea, UK, 1982. (PhD thesis.)

17 Green M, Isaac D H, Jenkins G M. Bone microstructure by collagenase etching. Biomaterials 1985; 6: 150-2.

18 Green M, Isaac D H, Jenkins G M. Collagen fibre orientation in bovine secondary osteons by collagenase etching. Biomaterials 1987; 8: 427-32.

19 Green M, Isaac D H, Jenkins G M. Mineral structure and preferred orientation in the fin bones of the plaice, Pleuronectes platessa. Biomaterials 1988; 9: 319-23.

20 Green M, Isaac D H, Jenkins G M. The structure of bone mineral and its spatial relationship with the collagen. 1987 Advances in Bioengineering 1987: 59-60. (ASME.)

21 Nokes L D M. Green M, Knight B. The use of scanning electron microscopy in the dating of human skeletal remains. J Forensic Sci Soc 1987; 27: 413-6.

22 Mackie I G, Green M, Clarke H, Isaac D H. Human bone microstructure by collagenase etching. J Bone Joint Surg (in press). 